\title{
Immediate effects of hybrid assistive limb gait training on lower limb function in a chronic myelopathy patient with postoperative late neurological deterioration
}

\author{
Shigeki Kubota ${ }^{1 *} \mathbb{D}$, Hideki Kadone ${ }^{2}$, Yukiyo Shimizu ${ }^{3}$, Masao Koda ${ }^{1}$, Hiroshi Takahashi ${ }^{1}$, Kousei Miura', \\ Fumihiko Eto ${ }^{1}$, Takeo Furuya ${ }^{4}$, Yoshiyuki Sankai ${ }^{5}$ and Masashi Yamazaki ${ }^{1}$
}

\begin{abstract}
Objective: The Hybrid Assistive Limb (HAL) has recently been used to treat movement disorders. Although studies have shown its effectiveness for chronic myelopathy, the immediate effects of HAL gait training on lower limb function have not been clarified. We conducted HAL gait training and examined its immediate effects on a 69-year-old man with re-deterioration of myelopathy in the chronic phase after surgery for compression myelopathy. The HAL intervention was performed every 4 weeks for 10 total sessions. Immediately before and after each session, we analyzed the patient's walking ability using the 10-m walk test. In the 4th HAL session, the gastrocnemius muscle activity was measured bilaterally using a synchronized motion capture-electromyogram system.

Results: The training effects became steady after the 2nd session. In sessions 2-10, the step length increased from 0.56 to $0.63 \mathrm{~m}$ (mean: $0.031 \mathrm{~m}$ ) immediately after HAL training. The motion capture-electromyogram analyses showed that considerable amounts of gastrocnemius muscle activity were detected during the stance and swing phases before HAL training. During and immediately after HAL training, gastrocnemius activity during the swing phase was diminished. HAL gait training has an immediate effect for inducing a normal gait pattern with less spasticity in those with chronic myelopathy.
\end{abstract}

Keywords: Wearable electronic devices, Gait, Exercise therapy, Spinal cord diseases

\section{Introduction}

The Hybrid Assistive Limb $\left(\mathrm{HAL}^{\circledR}\right)$, a wearable exoskeleton robot, can assist in the voluntary control of knee and hip joint motions $[1,2]$. The HAL provides physical support according to the wearer's voluntary intention by detecting his/her electrical signals, including muscle activity. Patients with spinal cord disorders cannot move their bodies because intention signals from the brain are

\footnotetext{
*Correspondence: s-kubota@md.tsukuba.ac.jp

${ }^{1}$ Department of Orthopaedic Surgery, Faculty of Medicine, University

of Tsukuba, 1-1-1 Tennodai, Tsukuba, Ibaraki 305-8575, Japan

Full list of author information is available at the end of the article
}

not appropriately transmitted from the injured spinal cord to the more distant body parts. The HAL captures faint myoelectrical signals in the periphery, and the HAL power unit generates sufficient assist torque by amplifying the patient's diminished joint torque. Thus, HAL can provide motion support for patients with spinal cord disorders. Previous studies have shown the effectiveness of HAL gait training for several spinal conditions, including spinal cord injury [3], acute and chronic stages after surgery for compression myelopathy [4-6], arteriovenous malformation [7], and spinal cord infarction [8]. However, there is no report on the immediate effect of HAL gait training. We conducted HAL gait training 
for a patient with re-deterioration of myelopathy in the chronic phase after surgery and examined the immediate effects of HAL gait training.

\section{Main text}

\section{Patient}

A 69-year-old man presented with late deterioration of myelopathy following spine surgery and underwent HAL gait training to improve his ability to walk. The progression of the gait dysfunction is summarized in Fig. 1.

\section{Preoperative clinical data}

The patient initially presented with clumsiness of his right hand and rapidly progressing gait disturbance. Imaging findings revealed compression myelopathy due to cervical ossification of the posterior longitudinal ligament (OPLL) and thoracic ossification of the ligamentum flavum (OLF) (Additional file 1).

\section{Surgery and postoperative course}

Posterior decompression surgery was performed for the compressed spinal cord. We performed laminoplasty at C4-C6, laminotomy of the inferior portion of C3 and cranial portion of C7, and laminectomy at T3-T8. Postoperative imaging findings showed that adequate decompression of the spinal cord was achieved at the thoracic spine, but slight compression of the cervical spinal cord remained at the $\mathrm{C} 3-\mathrm{C} 4$ level (Additional file 2). Postoperatively, the myelopathy was relieved. However, 8 years and 6 months after surgery, he experienced deterioration of his gait, although no worsening of upper extremity function was noted (Fig. 1). He experienced an increase in spasticity in the right leg. Analyses of magnetic resonance (MR) images showed atrophic changes in the spinal cord at the C4-C5 level (Additional file 3).

\section{HAL intervention}

Ten years and 11 months after surgery, the patient underwent HAL gait training. Approximately every 4 weeks, he underwent HAL gait training and completed 10 sessions (Fig. 1). The HAL intervention was provided by a medical doctor, who was present in case of emergency, as well as a therapist, two assistants, and an engineer, as reported previously $[6,9]$. Each HAL session lasted $60 \mathrm{~min}$, including the time for attaching/detaching the device, rest, and walking along a $25-\mathrm{m}$-long circuit several times. The net gait training time was approximately $20 \mathrm{~min}$. The patient did not use any assistive walking devices, such as a t-cane, lateral crutch, or walker in his daily life and during the HAL training. In the HAL session, the operator adjusted the degree of physical support provided to the patient. Table 1 shows the assist settings for the patients' hip and knee joints in each HAL session. Higher values of the HAL assist setting indicate greater physical support provided to the patient.

\section{Functional evaluation}

A 10-m walk test and 2-min walk test were conducted before (baseline) and after (after training) the HAL intervention. The initial testing (baseline) was performed 24 days before the 1st HAL session. The final test (after training) was performed 5 days after the 10th HAL session. During the $10-\mathrm{m}$ walk test, the patient was instructed to walk without wearing the HAL on a flat surface at a self-selected comfortable pace. Walking speed $(\mathrm{m} / \mathrm{min})$, step length $(\mathrm{m})$, and cadence (steps/min) were calculated as previously described $[6,9]$. During the 2 -min walk test, the patient was asked to walk for 2 min at a chosen maximal pace, and the total distance walked was recorded. The 10-m walk test was also performed in each HAL session just before and after detaching the HAL to evaluate the immediate effects of the HAL training on lower limb functions.

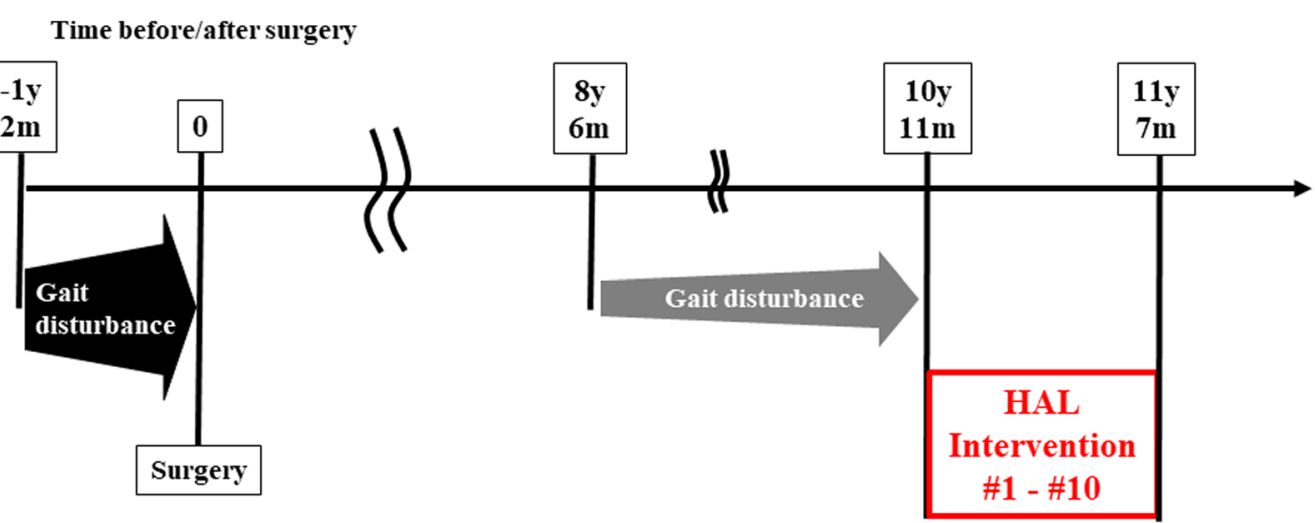

Fig. 1 The patient's symptom progression and the point in time at which Hybrid Assistive Limb training was performed 
Table 1 Assist setting for the patient's hip and knee joints at each HAL session

\begin{tabular}{|c|c|c|c|c|c|c|c|c|}
\hline \multirow[t]{3}{*}{ HAL session } & \multicolumn{4}{|l|}{ Hip } & \multicolumn{4}{|l|}{ Knee } \\
\hline & \multicolumn{2}{|c|}{ Right } & \multicolumn{2}{|l|}{ Left } & \multicolumn{2}{|l|}{ Right } & \multicolumn{2}{|l|}{ Left } \\
\hline & Flex & Ext & Flex & Ext & Flex & Ext & Flex & Ext \\
\hline 1 & 8.4 & 12 & 10 & 10 & 12 & 12 & 7 & 10 \\
\hline 2 & 6 & 10 & 5.4 & 9 & 10 & 10 & 5.4 & 9 \\
\hline 3 & 6 & 10 & 5.4 & 9 & 7 & 10 & 2.7 & 9 \\
\hline 4 & 4 & 10 & 5.4 & 9 & 10 & 10 & 6.3 & 9 \\
\hline 5 & 3 & 10 & 2.7 & 9 & 10 & 10 & 6.3 & 9 \\
\hline 6 & 5 & 10 & 4.5 & 9 & 10 & 10 & 6.3 & 9 \\
\hline 7 & 5 & 10 & 4.5 & 9 & 10 & 10 & 6.3 & 9 \\
\hline 8 & 5 & 10 & 4.5 & 9 & 10 & 10 & 6.3 & 9 \\
\hline 9 & 5 & 10 & 4.5 & 9 & 10 & 10 & 6.3 & 9 \\
\hline 10 & 5 & 10 & 4.5 & 9 & 10 & 10 & 6.3 & 9 \\
\hline
\end{tabular}

The assist setting is expressed as the value on a scale of $0-20$.

Flex, flexion; Ext, extension

Gait characteristics were measured using a VICON motion capture system (Vicon MX System with 16 T20s cameras; Oxford Metrics Ltd., Oxford, United Kingdom) three times during each session as follows: unassisted gait just before training with the robot (pre-HAL), gait while ambulating with the wearable robot during training (HAL), and unassisted gait after removal of the robot after training (post-HAL). During these tests, the patient was instructed to walk on a flat surface at a selfselected, comfortable pace. Auto-reflexive markers were attached to the feet following the VICON plug-in gait marker placement on the foot. The toe lift was computed according to the relative height displacement measured by the maximum height minus the minimum height of a toe marker for each step and then averaged among the extracted steps. The step length and toe lift were computed separately for the right and left sides, focusing on lateral symmetry.

The activity of the gastrocnemius muscle was recorded bilaterally using a Trigno Lab wireless electromyography (EMG) system (Delsys, United States). Because of the spasticity of the ankle plantar flexors, the patient had foot drop during the swing phase. Therefore, the phasedependent muscle activation of the plantar flexors was evaluated using the ratio of the integrated activity of the gastrocnemius during the swing phase to the activity over the entire step cycle (combined swing and stance phases).

\section{Results}

\section{Change in walking ability at baseline and after the HAL} intervention

Table 2 shows the data of the $10-\mathrm{m}$ and 2-min walk tests at baseline and after the HAL intervention.
Table 2 Walking ability at baseline and after the HAL intervention

\begin{tabular}{lcc}
\hline & At baseline & $\begin{array}{c}\text { After the HAL } \\
\text { intervention }\end{array}$ \\
\hline 10-m walk test & & \\
Speed (m/sec) & 1.06 & 1.31 \\
Step length (m) & 0.56 & 0.65 \\
$\quad$ Cadence (steps/min) & 114.6 & 121.9 \\
2-min walk test & & \\
$\quad$ Total walking distance $(\mathrm{m})$ & 142.5 & 156.6 \\
\hline
\end{tabular}

At baseline: data were obtained 24 days before the 1st HAL session

After the HAL intervention: data were obtained 5 days after the 10th HAL session

After the HAL intervention, the maximum hip angle flexion during the swing phase (mean $\pm \mathrm{SD}$ ) increased from $23.3 \pm 0.4^{\circ}$ to $32.5 \pm 1.2^{\circ}$ on the right side and from $27.6 \pm 0.8^{\circ}$ to $39.7 \pm 2.2^{\circ}$ on the left side (Additional file $4 \mathrm{~A}, \mathrm{~B})$. Similarly, maximum flexion decreased at the knee from $70.6 \pm 3.0^{\circ}$ to $63.7 \pm 3.3^{\circ}$ on the right side and from $65.8 \pm 1.7^{\circ}$ to $65.8 \pm 2.2^{\circ}$ on the left side (Additional file $5 \mathrm{~A}, \mathrm{~B})$.

\section{Change in walking ability immediately before and after HAL gait training in each HAL session}

Additional file 6 shows the data of the 10-m walk test immediately before and after the HAL gait training in each HAL session. The patient's gait speed after the HAL training was increased in sessions $2,3,5,7,8$, and 10 , did not change in sessions 4 and 6 , and decreased in sessions 1 and 9 (Additional file 6A). The step length did not change immediately before and after HAL training in session 1 . In sessions $2-10$, the step length was increased 
from $0.56 \mathrm{~m}$ to $0.63 \mathrm{~m}$ (mean: $0.031 \mathrm{~m}$ ) immediately after HAL training (Additional file 6B). The cadence slightly increased in session 7 and decreased in sessions $1-6$ and 8-10 (Additional file 6C).

\section{Results of kinematic motion analysis for the 4th HAL session}

The changes in the lower limb kinematics are shown in Additional file 7, 8, 9. Although step length increased from $0.51 \pm 0.03 \mathrm{~m}$ to $0.57 \pm 0.02 \mathrm{~m}$ on the right side, the left side was unchanged (from $0.59 \pm 0.02 \mathrm{~m}$ to $0.59 \pm 0.02 \mathrm{~m}$ ) (Additional file 7). Additional file 8 shows data for the toe lift of the right and left legs. In the right leg, it was $7.6 \pm 0.8 \mathrm{~cm}$ before, $12.7 \pm 4.6 \mathrm{~cm}$ during, and $9.5 \pm 0.8 \mathrm{~cm}$ after the HAL training. In the left leg, it was $10.9 \pm 0.9 \mathrm{~cm}$ before, $11.0 \pm 2.4 \mathrm{~cm}$ during, and $12.4 \pm 1.1 \mathrm{~cm}$ after the HAL training (Additional file 8).

Surface EMG profiles of the gastrocnemius muscle during the swing and stance phases are shown in Additional file 9. The activity of the gastrocnemius muscle was evident in the right leg during the swing phase (Additional file 9A), but the activity in the left leg during the swing phase was diminished during and after HAL training. Additional file 9B shows the ratio of the integrated activity of the gastrocnemius muscle during the swing phase to the activity over the entire step cycle (combined swing and stance phases). In the right leg, it was $1.90 \pm 0.77 \mathrm{mV}$ before, $0.21 \pm 0.07 \mathrm{mV}$ during, and $0.37 \pm 0.13 \mathrm{mV}$ after the HAL training. In the left leg, it was $1.79 \pm 0.45 \mathrm{mV}$ before, $0.76 \pm 0.29 \mathrm{mV}$ during, and $0.60 \pm 0.15 \mathrm{mV}$ after the HAL training.

\section{Discussion}

\section{Pathophysiology of late neurological deterioration}

We have occasionally encountered late neurological deterioration after surgery for compression myelopathy despite repeated adequate decompression of the spinal cord. We previously reported two cases of late neurological deterioration after decompression surgery for myelopathy due to cervical OPLL and thoracic OLF [6, 9]. The myelopathy symptoms were relieved after surgery but began to deteriorate without any apparent cause at 2 years and 15 years after surgery, respectively. The T2-weighted MR images (T2WI) of the decompressed spinal cords were characteristic. The spinal cords were markedly decompressed, but high-intensity changes appeared inside the spinal cord, which was atrophic.

We previously reported an autopsy case [10] in which laminoplasty for cervical spondylotic myelopathy was performed 9 years before the autopsy. T2WI showed a high-intensity area having a "snake-eyed" appearance at the decompressed spinal cord. Although such abnormal findings were predominantly observed in the gray matter by the MR image analyses, subsequent histopathological analyses demonstrated that atrophic changes in the spinal cord were evident in both the gray and white matter. Considering this and previous studies that analyzed the histopathology of damaged spinal cords in patients with compression myelopathy $[11,12]$, we suggest that, in the damaged spinal cord showing such "snake-eyed" appearance by MR image analyses, demyelination of the funiculus may progress in the white matter, and the spinal cord is in a condition of atrophy.

In the present case, the patient had myelopathy symptoms in both the upper and lower extremities preoperatively, and his symptoms were especially severe in his right leg. After surgery, the symptoms in both the upper and lower extremities were relieved. Although atrophic changes in the spinal cord existed at the C4-C5 level based on MR image analyses, late neurological deterioration developed only in his right leg. This suggests that, in the present case, atrophic changes in the white matter, such as the demyelination of the funiculus, existed at the C4-C5 level and may have participated in the development of the late neurological deterioration.

To date, there is no effective treatment for such late neurological deterioration caused by spinal cord atrophy. We previously performed gait training with HAL for late neurological deterioration after surgery for compression myelopathy, and obtained considerable recovery of gait function $[6,9]$. Thus, in the present case, we chose HAL gait training for the recovery of gait function.

\section{Effects of HAL gait training on lower limb function}

In the present case, the patient's walking ability was enhanced after 8 months of HAL intervention. The effect of HAL on improving gait in the present case was consistent with that of previous cases $[6,9]$, suggesting that repeated and long-term HAL gait training may cause the recovery of lower limb function in patients with chronic myelopathy. We further analyzed the HAL effect using motion capture and wireless EMG systems. Because the present patient showed severe paralysis in his right leg, we set a stronger assist level on the right side. Thus, the effect of HAL was detected predominantly in the right leg. The height of the toe lift was greater during the HAL gait training than before the HAL training (Additional file 8). Although the HAL does not have the function of assisting ankle motion, it can increase hip flexion and knee extension during training. This may be one of the reasons why toe lift increased during the HAL gait training. In addition, during the training, contraction of the gastrocnemius muscle decreased during the swing phase (Additional file 9), possibly also contributing to increased toe lift. 
Notably, the increased toe lift and decreased contraction of the gastrocnemius muscle during the swing phase were maintained immediately after the HAL training. We suggest that exercise memory during the HAL training was retained in the patient's brain, and he was able to reproduce the improved gait pattern after the HAL training even without HAL. It is possible that his brain relearned the normal gait pattern by the voluntary walking exercise with HAL.

\section{Limitations}

This study is a single case report and could not compare the efficacy of the HAL training with that of conventional rehabilitation.

\section{Abbreviations}

EMG: Electromyography; HAL: Hybrid assistive limb; MR: Magnetic resonance; OLF: Ossification of the ligamentum flavum; OPLL: Ossification of the posterior longitudinal ligament; T2Wl: T2-weighted MR images.

\section{Supplementary Information}

The online version contains supplementary material available at https://doi. org/10.1186/s13104-022-05979-4.

Additional file 1: Reconstruction images from computed tomography (CT) myelography of the cervical and thoracic spine before surgery. Midsagittal reconstruction CT myelogram of the cervical spine (A) and axial CT image at the $C 5$ level $(B)$ reveal segmental ossification of the longitudinal ligament (OPLL) at the C4-C6 vertebrae. The asterisks in (A) and (B) indicate the C5 OPLL. The spinal cord was compressed from the anterior and posterior directions at the C3-C4 and C4-C5 levels (red arrows). Midsagittal reconstruction CT myelogram of the thoracic spine (C) and axial CT image at the T4-T5 level (D) reveal multilevel ossification of the ligamentum flavum (OLF) at the T3-T8 vertebrae. The asterisks in (C) and (D) indicate the T4-T5 OLF. The spinal cord was compressed posteriorly at the T4-T5 level (blue arrow).

Additional file 2: Magnetic resonance (MR) images of the cervical and thoracic spine 14 months after surgery. T1-weighted (A) and T2-weighted (B) midsagittal MR images show sufficient decompression of the spinal cord at the thoracic spine. Magnification of the T2-weighted image at the cervical spine area (C) shows slight posterior compression of the spinal cord at the C3-C4 level (red arrow).

Additional file 3: MR images of the cervical spine 10 years and 6 months after surgery. T1-weighted (A) and T2-weighted (B) midsagittal MR images show slight posterior compression of the spinal cord at the C3-C4 level (red arrows). In the T2-weighted MR image (B), a high-intensity area was present inside the spinal cord at the C4-C5 level (red arrowhead), although the cord was thoroughly decompressed at this level. A T2-weighted MR axial image at the C4-C5 level (C) shows that the highintensity area was predominant in the gray matter with a "snake-eyed" appearance (blue arrowheads).

Additional file 4: Data of the kinematic motion analysis of the hip joint for HAL session 4. (A) Temporal profile of the angular position of the hip joint over the gait cycle (A) and range of motion of the hip over the gait cycle (B), measured without, immediately before, and after the HAL training. Error bars indicate standard error of the mean. Flex, flexion; Ext, extension; Pre, Pre-HAL training; Post, Post-HAL training; ROM, range of motion.

Additional file 5: Data of the kinematic motion analysis of the knee joint for HAL session 4. (A) Temporal profile of the angular position of the knee joint over the gait cycle $(A)$ and range of motion of the knee over the gait cycle (B), measured without, immediately before, and after the HAL training. Error bars indicate standard error of the mean. Flex, flexion; Ext, extension; Pre, Pre-HAL training; Post, Post-HAL training; ROM, range of motion.

Additional file 6: The 10- $\mathrm{m}$ walk test data immediately before and after HAL gait training, sessions 1-10. Gait speed (A), step length (B), and cadence (C).

Additional file 7: Data of the kinematic motion analysis of step length for HAL session 4. Step length of the right and left legs immediately before and after HAL training. Pre, Pre-HAL training; Post, Post-HAL training.

Additional file 8: Data of the kinematic motion analysis of toe clearance for HAL session 4. Toe lift of the right and left legs before, during, and after HAL gait training. The toe lift indicates toe clearance. Pre, Pre-HAL training; Post, Post-HAL training.

Additional file 9: Kinematic motion analysis using the VICON motion capture system and surface electromyography (HAL session 4). (A) Surface electromyography of the gastrocnemius muscles of the right and left legs during the stance and swing phases. (B) Gastrocnemius stance ratio of the right and left legs before, during, and after the HAL gait training. The gastrocnemius stance ratio indicates the muscle activation ratio of the gastrocnemius (swing phase to the total step cycle). Pre, Pre-HAL training; Post, Post-HAL training.

\section{Acknowledgements}

We would like to thank Mayuko Sakamaki and Yumiko Ito, Center for Innovative Medicine and Engineering (CIME), University of Tsukuba Hospital, for their excellent technical assistance.

\section{Authors' contributions}

SK and HK collected, analyzed, and interpreted the data; SK, HK, and MY wrote the first draft of the manuscript. SK also administered HAL therapy and collected clinical scores. MY organized the study. YUS, KM, and FE supported HAL therapy. MK, HT, and TF provided important comments on the planning and implementation of HAL treatment. YOS originally developed the robot suit HAL and conceived the idea of HAL therapy. All authors read and approved the final manuscript.

\section{Funding}

This work was supported by the Industrial Disease Clinical Research Grants from the Ministry of Health, Labour, and Welfare of Japan (14060101-01).

\section{Availability of data and materials}

All data generated or analyzed during this study are included in this published article [and its additional files].

\section{Declarations}

\section{Ethics approval and consent to participate}

The patient received a personalized explanation of the study, participation, and data usage before signing an informed consent form. This study was approved by the ethics committee of the Tsukuba University Faculty of Medicine.

\section{Consent for publication}

The patient provided written consent for publication of this case report and associated images.

\section{Competing interests}

Yoshiyuki Sankai is a founder, shareholder, and the CEO of CYBERDYNE, Inc. CYBERDYNE was not involved in the study design, data collection, analysis, writing, or submission of this article.

\section{Author details}

${ }^{1}$ Department of Orthopaedic Surgery, Faculty of Medicine, University of Tsukuba, 1-1-1 Tennodai, Tsukuba, Ibaraki 305-8575, Japan. ${ }^{2}$ Center for Innovative Medicine and Engineering, University of Tsukuba, 1-1-1 Tennodai, Tsukuba, Ibaraki 305-8575, Japan. ${ }^{3}$ Department of Rehabilitation Medicine, Faculty of Medicine, University of Tsukuba, Ibaraki 305-8575, Japan. 
${ }^{4}$ Department of Orthopaedic Surgery, Chiba University Graduate School of Medicine, Chiba 260-8677, Japan. ${ }^{5}$ Faculty of Engineering, Information and Systems, University of Tsukuba, 1-1-1 Tennodai, Tsukuba, Ibaraki 305-8573, Japan.

Received: 20 October 2021 Accepted: 18 February 2022

Published online: 04 March 2022

\section{References}

1. Kawamoto H, Sankai Y. Power assist method based on Phase Sequence and muscle force condition for HAL. Adv Robot. 2005;19(7):717-34. https://doi.org/10.1163/1568553054455103.

2. Sankai Y, Sakurai T. Exoskeletal cyborg-type robot. Sci Robot. 2018;3(17):eaat3912. https://doi.org/10.1126/scirobotics.aat3912.

3. Kubota S, Nakata Y, Eguchi K, Kawamoto H, Kamibayashi K, Sakane M, et al. Feasibility of rehabilitation training with a newly developed wearable robot for patients with limited mobility. Arch Phys Med Rehabil. 2013:94(6):1080-7. https://doi.org/10.1016/j.apmr.2012.12.020.

4. Kubota S, Abe T, Kadone H, Shimizu Y, Funayama T, Watanabe H, et al. Hybrid assistive limb (HAL) treatment for patients with severe thoracic myelopathy due to ossification of the posterior longitudinal ligament (OPLL) in the postoperative acute/subacute phase: a clinical trial. J Spinal Cord Med. 2019;42(4):517-25. https://doi.org/10.1080/10790268.2018. 1525975.

5. Soma Y, Kubota S, Kadone H, Shimizu Y, Takahashi H, Hada Y, et al. Hybrid assistive limb functional treatment for a patient with chronic incomplete cervical spinal cord injury. Int Med Case Rep J. 2021;2021(14):413-20. https://doi.org/10.2147/IMCRJ.S306558.eCollection.

6. Kubota S, Abe T, Kadone H, Fujii K, Shimizu Y, Marushima A, et al. Walking ability following hybrid assistive limb treatment for a patient with chronic myelopathy after surgery for cervical ossification of the posterior longitudinal ligament. J Spinal Cord Med. 2019;42(1):128-36. https://doi.org/10. 1080/10790268.2017.1313932.

7. Shimizu Y, Nakai K, Kadone H, Yamauchi S, Kubota S, Ueno T, et al. The Hybrid Assistive Limb ${ }^{\circledR}$ intervention for a postoperative patient with spinal dural arteriovenous fistula and chronic spinal cord injury: a case study. J Spinal Cord Med. 2018;41(6):710-7. https://doi.org/10.1080/10790268. 2017.1329916.

8. Watanabe H, Marushima A, Kawamoto H, Kadone H, Ueno T, Shimizu $Y$, et al. Intensive gait treatment using a robot suit hybrid assistive limb in acute spinal cord infarction: report of two cases. J Spinal Cord Med. 2019;42(3):395-401. https://doi.org/10.1080/10790268.2017.1372059.

9. Taketomi M, Shimizu Y, Kadone $H$, et al. Hybrid assistive limb $\left(\mathrm{HAL}^{\circledR}\right)$ intervention in a patient with late neurological deterioration post-thoracic myelopathy surgery due to ossification of the ligamentum flavum. Case Rep Orthop. 2018. https://doi.org/10.1155/2018/6171760.

10. Someya Y, Koda M, Hashimoto M, Okawa A, Masaki Y, Yamazaki M. Postmortem findings in a woman with history of laminoplasty for severe cervical spondylotic myelopathy. J Spinal Cord Med. 2011;34(5):523-6. https://doi.org/10.1179/107902611X13069205199503.

11. Ito T, Oyanagi K, Takahashi H, Takahashi HE, Ikuta F. Cervical spondylotic myelopathy. Clinicopathologic study on the progression pattern and thin myelinated fibers of the lesions of seven patients examined during complete autopsy. Spine (Phila Pa 1976). 1996;21(7):827-33. https://doi. org/10.1097/00007632-199604010-00010.

12. Guest JD, Hiester ED, Bunge RP. Demyelination and Schwann cell responses adjacent to injury epicenter cavities following chronic human spinal cord injury. Exp Neurol. 2005;192(2):384-93. https://doi.org/10. 1016/j.expneurol.2004.11.033.

\section{Publisher's Note}

Springer Nature remains neutral with regard to jurisdictional claims in published maps and institutional affiliations.
Ready to submit your research? Choose BMC and benefit from:

- fast, convenient online submission

- thorough peer review by experienced researchers in your field

- rapid publication on acceptance

- support for research data, including large and complex data types

- gold Open Access which fosters wider collaboration and increased citations

- maximum visibility for your research: over $100 \mathrm{M}$ website views per year

At BMC, research is always in progress.

Learn more biomedcentral.com/submissions 